\title{
Posgrados: visión y percepción en la Universidad Pedagógica y Tecnológica de Colombia (UPTC) ${ }^{1}$
}

\author{
Doris Lilia Torres Cruz ${ }^{2}$ \\ Universidad Pedagógica y Tecnológica de Colombia \\ doris.torres@uptc.edu.co \\ Jinny Fernanda Prieto Ortega ${ }^{3}$ \\ Grupo de investigación LEEN \\ jinnyta_ona@hotmail.com
}

Recepción: 19/09/2013

Evaluación: 23/11/2013

Aceptación: 13/12/2013

Artículo de Reflexión

DOI: http:/ / dx.doi.org/ 10.9757/Rhela.22/12

\section{RESUMEN}

Este artículo tiene como objetivo socializar los resultados parciales del proyecto de investigación titulado "Leer y escribir la formación posgraduada con un lenguaje de responsabilidad social", registrado en el Sistema General de Investigaciones bajo el número 1289. Identificar las percepciones de los estudiantes de pregrado y posgrado frente a los programas de formación posgraduada de la Facultad de Ciencias de la Educación permite puntualizar la relevancia de los procedimientos implementados por medio de la política educativa institucional y la normatividad vigente. Se asume la investigación desde una perspectiva histórica hermenéutica, por medio de la cual se da un proceso diacrónico en la creación de programas de posgrado en la Facultad de Ciencias de la Educación-UPTC (Universidad Pedagógica y Tecnológica de Colombia) como una de las pioneras en la formación de maestros en el país, además de un enfoque mixto de análisis cualitativo y cuantitativo de los resultados encontrados. En un primer momento, se dan a conocer los hallazgos de la encuesta piloto, luego el análisis a las encuestas realizadas a estudiantes de los programas de pregrado. En un tercer momento se describen las percepciones de estudiantes de posgrado de la misma facultad. Finalmente, se presentan las conclusiones frente a los procesos del nivel posgraduado, su política de internacionalización, investigación, articulación con la Educación Media y Técnica, junto con la transferencia de conocimiento con criterios de calidad y equidad social.

Palabras clave: Revista Historia de la Educación Latinoamericana, posgrados, políticas, calidad educativa, Estado.

1 Este artículo recoge los resultados parciales del Proyecto de Investigación "Leer y escribir la formación posgraduada. Un lenguaje de responsabilidad social" SGI 1289, UPTC.

2 Doctora en Ciencias de la Educación, RUDECOLOMBIA, Magíster en Lingüística de la Universidad del Valle, Licenciada en Idiomas modernos de la UPTC, Docente de la UPTC, coordinadora del grupo de investigación Lenguajes en educación, LEEN, Coordinadora Subsede cátedra UNESCO para el mejoramiento de la calidad y equidad de la educación en América Latina, sus trabajos de investigación giran en torno a Lenguajes en Educación.

3 Licenciada en Ciencias Sociales e integrante del grupo de investigación Lenguajes en educación, LEEN. 


\author{
Postgraduate Programs: Vision and perception \\ in the Universidad Pedagógica y Tecnológica de \\ Colombia (UPTC)
}

\author{
Pós-graduação: Visão e percepção na Univer- \\ sidade Pedagógica e Tecnológica da Colômbia \\ (UPTC)
}

This article aims at socializing the partial results of a research project entitled to read and to write the graduate development with a language of social responsibility, recorded in the General Research System with the number 1289. Identifying the undergraduate and postgraduate students' perceptions face the postgraduate programs in the Faculty of Educational Sciences. This allows to point out the relevance of the procedures implemented through the educational policy and institutional regulations. The research is assumed from a historical and hermeneutic perspective, through this there is a diachronic process in the creation of postgraduate programs in the faculty of Sciences of Education-UPTC (Universidad Pedagógica y Tecnológica de Colombia). This University as one of pioneers in the teachers development in the country. Therefore, it has a mixed approach of qualitative and quantitative analysis of found data.

In a first step, the findings of the pilot survey; then, the analysis to the surveys of students of the undergraduate programs; for in a third time, describe their perceptions of graduate students from the same faculty. Finally, conclusions are presented in front of the processes of the postgraduate level, its policy of internationalization, research and links with the secondary and technical education, along with the transfer of knowledge with the criteria of quality and social equity.

Key words: postgraduate programs, policies, education quality, state.

\section{RESUMO}

Este artigo tem como objetivo socializar os resultados parciais do projeto de pesquisa intituladoLereescrever a formação pós-graduada com uma linguagem de responsabilidade social, registrado no Sistema Geral de Pesquisa sob o número 1289. Identificar as percepções dos estudantes de graduação e pós-graduação frente aos programas de formação pós-graduada da Faculdade de Ciências da Educação, permite pontuar a relevância dos procedimentos implementados por meio da política educativa institucional e da normatividade vigente. A pesquisa se assume a partir de uma perspectiva histórico-hermenêutica, por meio da qual se dá um processo diacrônico na criação de programas de pós-graduação na Faculdade de Ciências da Educação - UPTC (Universidade Pedagógica e Tecnológica da Colômbia) como uma das pioneiras na formação de professores no país, ademais usando um enfoque misto de análise qualitativa e quantitativa dos resultados encontrados. Em um primeiro momento, se dão a conhecer os acertos da indagação piloto; logo, a análise das questões realizadas a estudantes dos programas de graduação; para em um terceiro momento, descrever as percepções de estudantes de pós-graduação da mesma faculdade. Finalmente, se apresentam as conclusões frente aos processos do nível pós-graduado, sua política de internacionalização, pesquisa, articulação com a Educação Média e Técnica, junto com a transferência de conhecimento com critérios de qualidade e equidade social.

Palavras-chave: Revista História da Educação Latino-americana pós-graduação, políticas, qualidade educativa, Estado. 


\section{INTRODUCCIÓN}

Entrado el siglo XXI en Colombia, las políticas educativas tuvieron como objetivo transformar el sistema, en magnitud y pertinencia, para garantizar la competitividad del país y asegurar el mejoramiento de la calidad de vida de la población. A la luz de este propósito, se establecieron en el sector tres ejes de cobertura, calidad y eficiencia. Los cuales se sobre diagnosticaron con el fin de justificar la llamada Revolución Educativa. ${ }^{4}$ Se determinó que la educación era un factor estratégico y prioritario del desarrollo humano, social y económico de los países y un instrumento esencial para la construcción de sociedades más autónomas, justas y democráticas. Así mismo, se dijo que la cobertura y la calidad de la educación eran factores determinantes para garantizar la competitividad y que, a pesar de los esfuerzos realizados, Colombia no había logrado universalizar el acceso de niños y jóvenes a una educación de calidad. ${ }^{5}$

Los indicadores de cobertura, eficiencia y calidad del sistema educativo indicaban que los avances habían sido lentos e insuficientes y que en muchos casos los aumentos en cobertura se lograban a costa de la calidad. La falta de educación constituía uno de los factores esenciales en aumento de la pobreza y la persistencia de la desigualdad. La formación posgraduada no quedó exenta de estas condiciones y se le hacía un llamado a generar procesos más contundentes y significativos que apuntaran a la solución de las necesidades locales, con la respectiva transferencia de conocimiento. Así se exigió a los posgrados resultados de alto impacto social, dado que se entendían como la punta de lanza para el desarrollo y el avance de conocimiento en un siglo XXI en pleno auge. Para el año 2005, esta política se materializaba con la implementación de proyectos que apuntaban a una visión empresarial, donde los posgrados eran los llamados a liderar las políticas educativas y a proponer proyectos de inversión social de acuerdo con las necesidades y en alianza con los sectores productivos y económicos. ${ }^{6}$

4 Colombia. Ministerio de Educación Nacional. Acciones y Lecciones Revolución Educativa 2002-2010 (Bogotá: MEN, 2002), consultado el 25 de octubre de 2013 http://www.mineducacion.gov.co/Revolucioneducativa

5 Colombia. Ministerio de Educación Nacional, Acciones y Lecciones, 31.

6 La UPTC, con la Facultad de Ciencias de la Educación, lideró un proyecto titulado Fortalecimiento a la capacidad de gestión de los directivos docentes, coordinado por la Pontificia Universidad Javeriana, y donde la UPTC participó con la ejecución de este enfoque a 150 Instituciones Educativas de Boyacá (100), Arauca (30) y Casanare (20). 
Algunos cuestionamientos en contravía a esta tendencia planteaban que la materia prima no era bruta, ni depositaria de saberes dictatoriales, ni de verdades absolutas establecidas por poderes hegemónicos. ${ }^{7}$ Se volvió en muchas ocasiones a entender al individuo como un agente pasivo en el proceso educativo, sobre el cual se esperaba generar un producto de calidad. Las condiciones empresariales que se imponían desconocían la multiculturalidad del territorio y la diversidad de posibilidades de cobertura, calidad y eficiencia, frente a los requerimientos económicos internacionales. Así la formación posgraduada no quedó ajena a vincular en su discurso la necesidad de establecer alianzas entre la educación, la empresa y el Estado.

A pesar de las fuertes críticas por parte de directivos y docentes, se instalaron en el lenguaje académico y de formación posgraduada términos como gestión, articulación, indicadores, aprendizaje por procesos, competencias investigativas, logros, metas y medición de resultados. ${ }^{8}$ Se entendía, entonces, que un programa de posgrado en educación contribuiría a consolidar la formación tanto de directivos como de docentes en un ámbito empresarial capaz de liderar cuatro gestiones: académica, administrativa, financiera y comunitaria. ${ }^{9}$

Si bien, se pensaba que como docentes se estaba al servicio del conocimiento y que este pasaba por medio de diferentes objetos a los procesos sociales, permeando estructuras políticas, económicas y culturales que se superponían y daban cabida a la consolidación de proyectos hegemónicos establecidos por minorías políticas, religiosas o económicas sólidas, también se sabía que, el poder y la dominación de un grupo sobre el otro se mediaba por el control en el acceso al discurso; lo cual llevaba a concebir al discurso como una forma, no solo de control social sino también de control sobre las mentes de los interlocutores, es decir, sobre las representaciones de una educación como empresa. ${ }^{10}$

\footnotetext{
7 Así, para el año 2009, y los que siguieron, se implementaron proyectos como "Estrategia de acompañamiento a los Establecimientos educativos de bajo logro, para el fortalecimiento en su gestión escolar con un enfoque de atención inclusiva", a la fecha y después de tanta intervención un buen número de docentes sabían leer y escribir con un enfoque "de responsabilidad social".

8 Véase programas de formación posgraduada. Políticas de formación posgraduada, AUIP. Especialización en Gerencia Educacional. Creación de Programas en Colombia a comienzos del Siglo XX.

9 Véase perfil de egresados de los programas de posgrados de la Facultad de Ciencias de la Educación de la UPTC.

10 Teun Van Dijk, "Ideología y análisis del discurso". Revista Utopia y Praxis Latinoamericana 10 (2005): 9.
} 


\section{Visión histórica de los posgrados}

Para la UPTC, con domicilio en Tunja, el nivel posgraduado se gesta desde el momento mismo de su creación (decreto 2655 del 10 de Octubre de 1953). Si bien, buena parte del desarrollo histórico de la universidad ha estado enfocado en el nivel de pregrado, cabe destacar que solo en las dos últimas décadas del siglo XX, y después de la Ley 30 de 1992, es que se da un auge significativo de Especializaciones y Maestrías en el país. Sin embargo, a nivel doctoral únicamente a finales del siglo $\mathrm{XX}$, la universidad contaba con un programa denominado Doctorado en Ciencias de la Educación, el cual se reconoce como uno de los pioneros en la formación a este nivel con la red de Universidades regionales y estatales de Colombia, RUDECOLOMBIA.

Entrado el siglo XXI, la UPTC implementa significativamente la normatividad del nivel posgraduado y promueve la investigación y productividad académica como una de las directrices de acreditación institucional. Así, hoy en día se cuenta con cinco programas de doctorado, cuatro en Educación y uno en Ingeniería: Doctorado en Ciencias de la Educación, Doctorado en Geografía, Doctorado en Historia, Doctorado en Lenguaje y Cultura y Doctorado en Ingeniería en Ciencias de los Materiales. Las maestrías proliferaron en profundización e investigación, de acuerdo con el Decreto 1295 de 2010. Entrada la segunda década de este siglo, la creación y promoción de las Especializaciones disminuyó significativamente, dado el nivel de reconocimiento de estas en el ámbito social y económico.

La UPTC no fue ajena a la visión occidental de la formación posgraduada que surgió en Europa, la cual asumió procesos eclécticos gestados bien en Alemania, Francia, Rusia, Inglaterra o EEUU con el fin de fundamentar el desarrollo de la ciencia por medio de la investigación.

El modelo de la UPTC, es un modelo mixto y por lo tanto resulta difícil aspirar a conformar un modelo con características propias. Cada Facultad de la universidad trata de desarrollar un esquema acorde con sus necesidades académicas. En ese orden de ideas, se puede afirmar que la presencia de los posgrados en la UPTC fue el resultado de la formación de profesores en algunos de los países europeos o en Estados Unidos y quienes hicieron transferencias de dichos modelos a la universidad. En consecuencia, los primeros posgrados que se crearon obedecían más a los modelos extranjeros 
que al actuar con las necesidades regionales del Departamento y el país. Se está en presencia de un desarrollo desigual, que lleva a implementar mecanismos de cooperación con el objeto de solucionar los problemas económicos y sociales de la región. Lo anterior reafirma la propuesta de intensificar el proceso de internacionalización a nivel de estudios de posgrados en América Latina. La interacción no solamente sirve para el desarrollo económico y social sino también para el desarrollo de las ciencias, en la medida que los resultados de la investigación no solamente benefician al país que los produce sino a todos los países de la región. Lo anterior, en la medida que la educación posgraduada sea un factor decisivo para el desarrollo y el poder político en la actual sociedad del conocimiento. Por esto, no se concibe que una universidad limite el desarrollo de políticas que tienden a privilegiar el valor estratégico que tienen los estudios de posgrado, como elemento de punta en el avance de las condiciones de vida de los respectivos países.

La normatividad y los posgrados en la UPTC han marchado en manera paralela. Por un lado, la normatividad regula situaciones ya creadas y, por el otro, las prácticas desarrolladas en los posgrados sirven para modificar o crear la normatividad existente. La situación descrita se puede analizar no solo por medio de la evolución normativa sino por medio de la evolución histórica que han tenido los postgrados en la Universidad. ${ }^{11}$

El desarrollo de la UPTC no fue extraño al proceso que había tenido la universidad en Europa, en el imperio Español, a la evolución que había tenido el concepto de universidad, desde la edad media. ${ }^{12}$ La anterior precisión obedece al hecho que en el periodo colonial no hubo universidades en Brasil. Esta es una paradoja, dado que el desarrollo universitario en este país se da en el siglo $X X$, contrario a otros países iberoamericanos que contaron con universidades desde el siglo $\mathrm{XVI} ;{ }^{13}$ por ello no es exagerado presumir que se pueda dar un desarrollo acelerado de los programas de posgrados en los países de lengua española en Iberoamérica.

11 Doris Lilia Torres Cruz, "Postgrados en Iberoamérica”, VIII Congreso Internacional de la Sociedad de Historia de la Educación Latinoamericana, (Colombia, Manizales, 2011).

12 La primera universidad que se fundó en Iberoamérica fue la Universidad de Santo Tomas de Aquino en Santo Domingo que data de 1538, luego se fundaron universidades en Lima y en México. Las universidades fundadas a iniciativas de las órdenes religiosas datan principalmente del siglo XVII.

13 Diana Soto Arango "El profesor universitario de América Latina: Hacia una responsabilidad ética, científica y social", Revista de la Educación Latinoamérica 13 (2009): 166-188. 
Como paréntesis a esta visión, cabe resaltar que desde comienzos del siglo $X X,{ }^{14}$ ocurrió en los países latinoamericanos una cierta estabilidad política que permitió el desarrollo universitario con un sesgo profesionalizante, asumiendo el modelo francés y tomando distancia del modelo alemán. Este aspecto demoró el desarrollo de la universidad latinoamericana. ${ }^{15} \mathrm{Si}$ se hubiera aceptado desde el principio el paradigma germano, otro hubiera sido el desarrollo de los posgrados para la ciencia y la investigación en esta parte del mundo. La nueva universidad latinoamericana se basó en el movimiento de Córdoba de 1918, que tuvo, entre otros motivos, el promover una clase media emergente y una universidad autónoma; sin embargo, entre sus objetivos no se vio claramente el desarrollo de los estudios posgraduados y ese hecho ha marcado el imaginario de este nivel en América Latina. El movimiento estudiantil del 68, en París, recogió los frutos de la reforma de Córdoba, pero no se proyectó en el desarrollo de los estudios postgraduados. Es necesario distinguir que, inicialmente, correspondían a una élite reducida pero el interés por desarrollar este nivel surge solamente después de la mitad del siglo XX en América Latina, con posterioridad a la Segunda Guerra Mundial. Sin embargo, los avances no se registraron como consecuencia de la intención del desarrollo económico de la región sino más bien, para incrementar el prestigio de algunas entidades de educación superior y de quienes seguían los cursos. En esa medida, el impacto y las percepciones que se tienen en esta época proyectan el devenir histórico de este nivel. ${ }^{16}$

\section{Percepciones de estudiantes de pregrado y posgrado acerca de la formación posgraduada de la Facultad de Ciencias de la Educa- ción de la UPTC ${ }^{17}$}

Esta investigación buscó identificar y analizó algunas de las percepciones de la formación posgraduada de los estudiantes de la UPTC, Facultad de Ciencias de la Educación, con el fin de identificar las perspectivas que se construyen en torno a los programas de posgrados, en una época donde la innovación, investigación, internacionalización son sine quanon a la condición de estudiante universitario.

\footnotetext{
14 Doris Lilia Torres Cruz, "Textos y pedagogías en los albores del siglo XX en Colombia”, Revista Historia de la Educación Latinoamericana 16 (2011): 255- 278.

15 Soto, "El profesor universitario", 175.

16 Olga Yanet Acuña Rodríguez, "Tendencias historiográficas vistas a través de las tesis del Doctorado en Ciencias de la Educación RUDECOLOMBIA (1988-2007)”, Revista Historia de la Educación Latinoamericana 12 (2009): 79-95.

17 Los datos estadísticos en cada una de las gráficas son ponderaciones aproximadas. Se realizó el diseño sin posiciones decimales, las cuales determinan el formato utilizado dentro del análisis de todos los resultados presentados en este artículo.
} 
En un primer momento y al aplicar la encuesta piloto (Total 20 estudiantes: 6 mujeres y 14 hombres) de medición de impacto, a cuatro programas de pregrado de la Facultad de Ciencias de la Educación: Licenciatura en Ciencias Sociales, Licenciatura en Educación Física, Licenciatura en Lenguas Extranjeras y Licenciatura en Idiomas Modernos, se observó que el 52\% de los estudiantes manifestó conocer más de dos programas de posgrado, especialmente por la información de un amigo, o por un folleto y, en muy pocas ocasiones, por medio de la web, la radio o prensa escrita. De igual manera, en un número mínimo, dijeron conocer los programas por la socialización de los docentes en el aula de clase. Así mismo, el campo que se vio impactado favorablemente al cursar y finalizar un programa de posgrados fue el familiar, seguido por el social, pedagógico y de último el científico. Se observó que el $100 \%$ de los encuestados creen que quien cursa un posgrado sin duda mejorará su condición personal y académica, seguida por el aspecto económico y el estatus social. El 100\% de los encuestados contestó que se deberían hacer posgrados en el mismo campo del pregrado y en temas afines. Solo un reducido número de estudiantes (2) manifestó que en campos distintos. Finalmente, los encuestados manifestaron que era necesario mejorar la oferta de los programas de posgrado, la socialización permanente y continua de cada uno por medio de los medios masivos de comunicación, tanto a nivel nacional como internacional; disminuir los costos e incrementar el número de becas que fomenten la investigación y el desarrollo social; mejorar los mecanismos de ingreso a los programas de posgrado, con semilleros de investigación y jóvenes investigadores; así mismo, sugieren crear programas de posgrados a fines a todas las licenciaturas de la Facultad, en condiciones físicas adecuadas.

Fue interesante observar que un número significativo de estudiantes de pregrado desconocían los niveles de posgrado: especialización, maestría, doctorado; asumiendo todos los niveles como uno solo. Así mismo, se observó que aún no hay claridad sobre las diferencias con los eventos de extensión, los cuales, en varias ocasiones, fueron confundidos con un programa de posgrado.

En un segundo momento, se realizó el análisis de reconocimiento de los programas de posgrado por parte de los estudiantes de pregrado de la Facultad de Ciencias de la Educación de la UPTC, con una muestra aleatoria, se seleccionaron cinco estudiantes, cada uno de los programas 
de Licenciatura en Educación Física, Recreación y Deporte, Licenciatura en Idiomas Modernos, Licenciatura en Ciencias Sociales, Licenciatura en Artes Plásticas, Licenciatura en Psicopedagogía y Licenciatura en Matemáticas, con el objetivo de valorar el impacto y la visibilidad que el nivel de posgrado tiene a nivel de pregrado en el contexto académico de la Facultad de Ciencias de la Educación de la UPTC. Así, la variable independiente correspondió a estudiantes de pregrado de los semestres $6^{\circ}$ a $8^{\circ}$ para un total de treinta encuestados. El $66 \%$ de las encuestas se aplicaron dentro del aula de clase, con autorización por parte del docente responsable de la asignatura, lo cual favoreció a las preguntas abiertas en relación con las respuestas dadas. El $34 \%$ restante, se aplicó en los intermedios de clase y en el tiempo; este aspecto desfavoreció las posibles respuestas frente a las preguntas abiertas. Del total de encuestados, el $60 \%$ corresponde al género femenino y el $40 \%$ al género masculino, sin que se determine ningún porcentaje frente a otros géneros.

\section{Gráfica 1. Porcentaje de reconocimiento de los programas de pos- grado de la Facultad de Ciencias de la Educación de la UPTC, según estudiantes de pregrado}

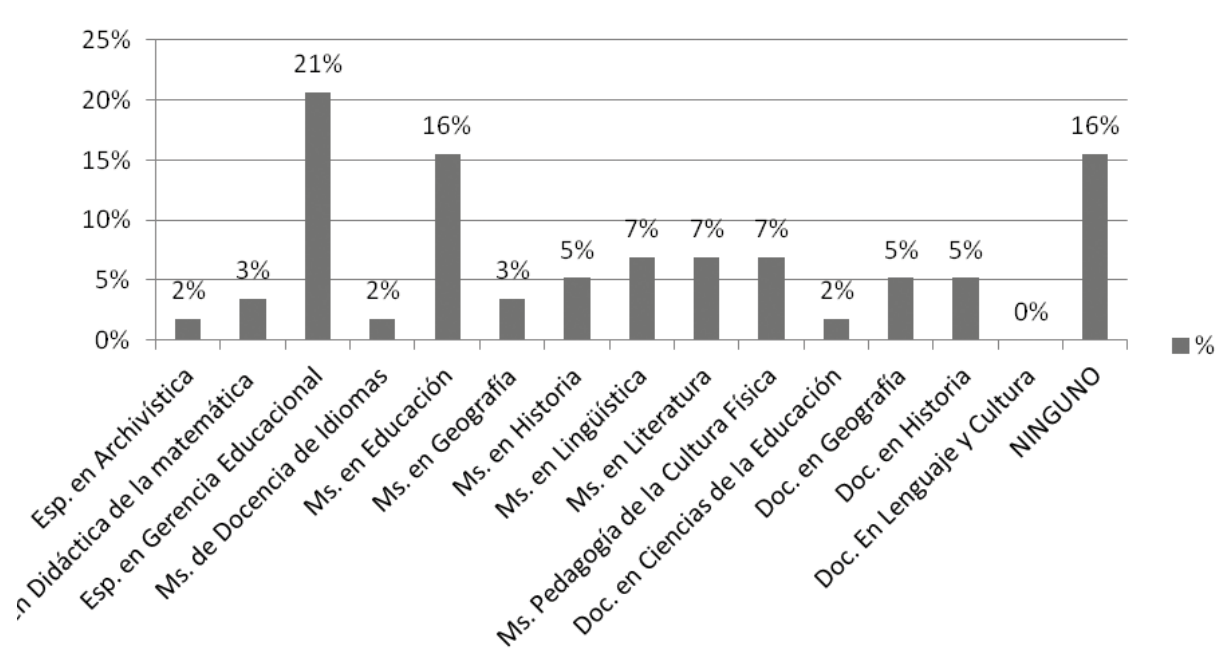

Fuente: Grupo de Investigación Lenguajes en Educación - LEEN, UPTC, 2013. 
En relación con el reconocimiento de los catorce programas de posgrado que ofrece la Facultad de Ciencias de la Educación, UPTC, (cuatro Doctorados, siete Maestrías y tres Especializaciones), los estudiantes de pregrado, en un $58 \%$ de las respuestas, identificaron los programas de posgrado propios de la Facultad, siendo la Especialización en Gerencia Educacional reconocida por el $21 \%$ de los encuestados, seguida por la Maestría en Educación en un $16 \%$, los demás programas como las maestrías en Lingüística, Literatura, Pedagogía de la Cultura Física estuvieron con un 7\%, respectivamente, seguido de las Maestrías en Historia con un 5\%, Maestría en Geografía y Especialización en Didáctica de la Matemática con un $3 \%$ cada una con un porcentaje de 2\%, respectivamente, para la Especialización en Archivística, Maestría en Docencia de Idiomas y Doctorado en Ciencias de la Educación y, finalmente, el Doctorado en Lenguaje y Cultura quedó sin identificación dentro de la opinión de los estudiantes de pregrado. (Gráfica 1). Lo anterior refleja el impacto de la normatividad como la Resolución 016 de 2010, por medio de la cual se autoriza como modalidad de grado, para el pregrado, los estudios de posgrado especialmente de una especialización o maestría. Este tema genera polémica en de los procesos de formación en investigación, por cuanto los estudiantes incursionan a nivel posgraduado sin la elaboración del trabajo monográfico y, en algunas ocasiones, sin el conocimiento básico sobre los parámetros mínimos en investigación. Los estudiantes acceden a la especialización con el fin de agilizar sus procesos de titulación y terminar en un semestre académico con los requisitos establecidos en el pregrado.

De igual manera, se observa que un número significativo de respuestas dadas por los encuestados, es decir en el $42 \%$ de ellas, ubican los programas de posgrado sin diferenciar las Facultades entre sí. Mencionan programas como Maestría en Derechos Humanos que corresponde a la Facultad de Derecho y Ciencias Sociales, lo mismo para la Especialización en Pedagogía de los Derechos Humanos de la seccional Duitama. Este aspecto permite entender que, a nivel posgraduado, se hace necesaria la apertura entre las Ciencias Sociales y las posibilidades de interacción entre un programa de pregrado y varios campos de acceso a nivel postgraduado. 


\section{Gráfica 2. Porcentaje medios de información que dan a conocer los programas de posgrado de la Facultad de Ciencias de la Edu- cación de la UPTC, según estudiantes de pregrado}

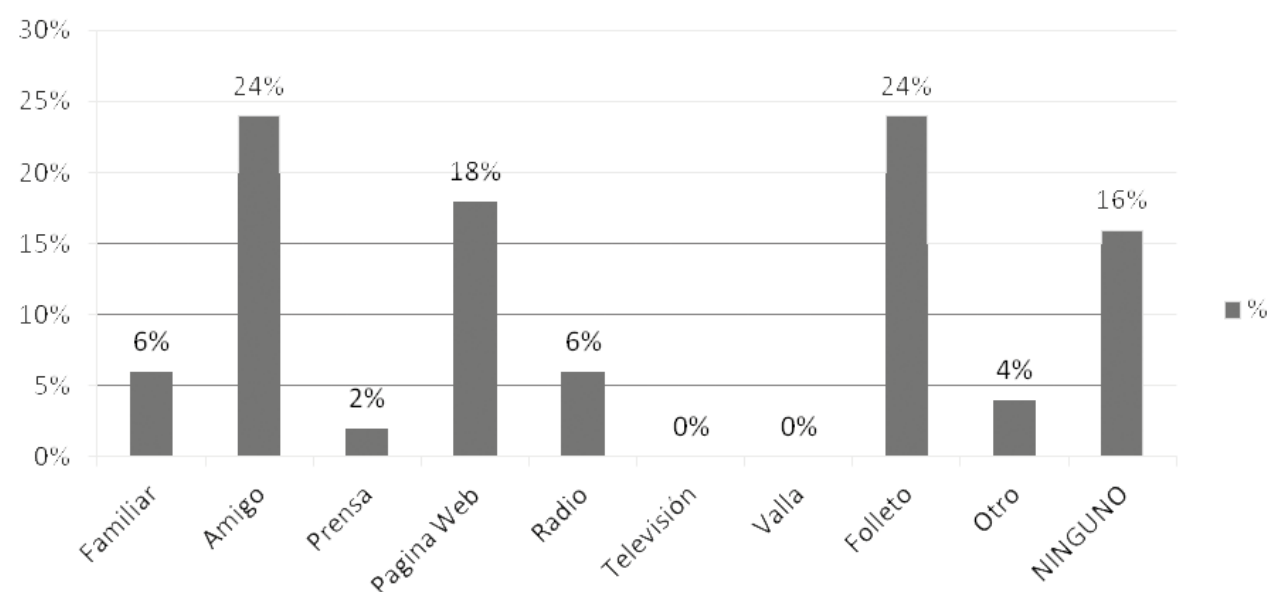

Fuente: Grupo de Investigación Lenguajes en Educación - LEEN, UPTC, 2013.

Así mismo, se encontró en este primer ítem que un porcentaje del 30\% del total de los encuestados perciben programas de posgrado que en la realidad no existen, o bien no se ofertan o corresponden a líneas de investigación, que están en proceso de creación, por ejemplo el Doctorado Historia de la Educación, Maestría Actividad Física Para la Salud, Docencia Universitaria, Lingüística Aplicada, Neuropedagogía, Trabajo Social y Recursos Humanos, Educación Especial, Doctorado en Derechos Humanos.

En relación con la identificación del instrumento que permite una difusión efectiva de los programas de posgrado de la Facultad de Ciencias de la Educación, se encontró que un $24 \%$, respectivamente, obedece principalmente a la socialización que se da entre amigos y por medio de folletos que editan los diferentes programas de posgrado. Lo cual corrobora el resultado de la encuesta piloto. Así mismo, un 18\% manifestó conocer los programas de posgrado por medio de la página web de la Universidad; seguido de la radio y el medio familiar cada uno con un 6\%; para dejar en un $2 \%$ a la prensa, tanto local como nacional. Es necesario destacar que el $16 \%$ manifestó no conocer, por ningún medio, los programas de posgrado. La televisión y valla publicitaria dieron lugar a un $0 \%$ para cada una, como se observa en la gráfica 2. 
En consecuencia, es prioritaria la necesidad de ejercer un mayor impacto y socialización de los posgrados a nivel nacional e internacional. Se percibe que el conocimiento es local y se desperdician los recursos y las plataformas de visibilidad a las cuales podrían acceder los programas de posgrado, como las fuentes masivas de información, tanto prensa, radio, televisión, web, entre otros. Se encontró que existe una socialización mínima del $4 \%$ por parte de docentes y de eventos, como congresos o seminarios hacia los programas de Postgrado.

De acuerdo con la gráfica 3, la percepción frente al campo que se ve impactado favorablemente al cursar y finalizar un programa de posgrado de la Facultad de Ciencias de la Educación de la UPTC, el 33\% optó por el campo personal, seguido por el social con un $21 \%$, el institucional con el 16\%, el familiar con el $13 \%$, el científico con un $11 \%$ y el político con $5 \%$. Es interesante percibir que a pesar de promover la investigación como un elemento fundamental para el avance de la Ciencia, sea este campo reconocido con un bajo impacto para los estudiantes de pregrado y, en cambio, sí se orienta hacia la búsqueda de la consolidación personal por encima de lo social y familiar.

\section{Gráfica 3. Porcentaje de impacto al cursar y finalizar un programa de posgrado de la Facultad de Ciencias de la Educación de la UPTC, según estudiantes de pregrado}

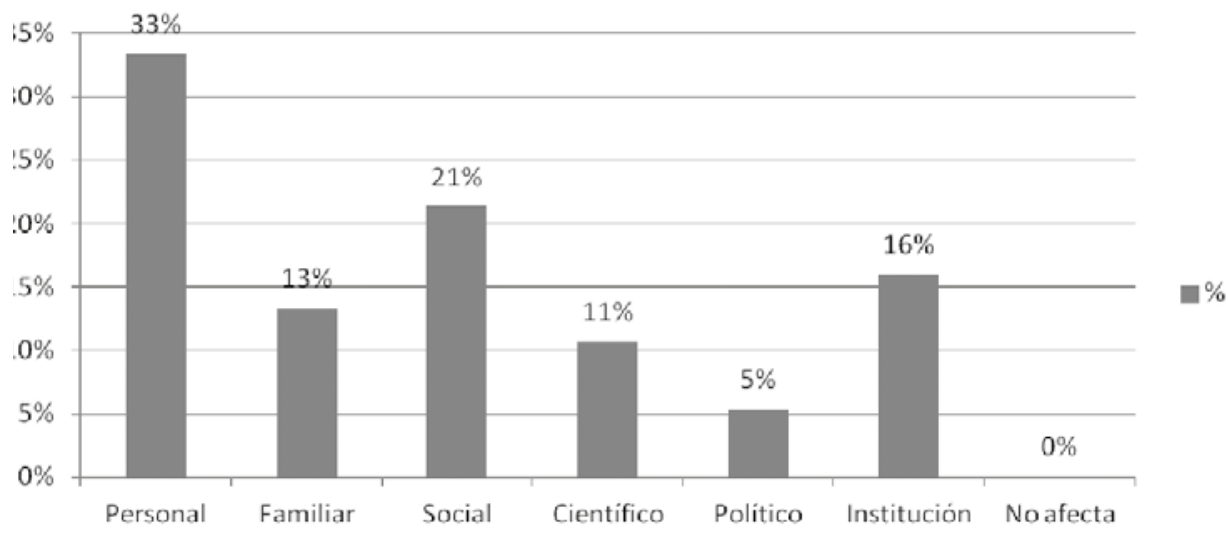

Fuente: Grupo de Investigación Lenguajes en Educación - LEEN, UPTC, 2013 
Continuando, en el análisis de la gráfica 4, los estudiantes de pregrado perciben que la formación posgraduada apunta hacia la construcción de sí mismo sobre el deber colectivo. Sin duda, se encuentra que quien cursa un programa de posgrado mejorará en su calidad académica con un $47 \%$, seguido por un $28 \%$ en su salario, el $17 \%$ en estatus, y el $9 \%$ la vida familiar. Sumando los porcentajes, el estatus, el salario y la vida familiar corresponderían a un $54 \%$, por encima de la calidad académica. Es decir que, aún existe la percepción de buscar un reconocimiento por medio del título, más que procesos de formación en investigación e innovación.

\section{Gráfica 4. Porcentaje de los aspectos que mejorarán al cursar un programa de posgrado de la Facultad de Ciencias de la Edu- cación de la UPTC, según estudiantes de pregrado}

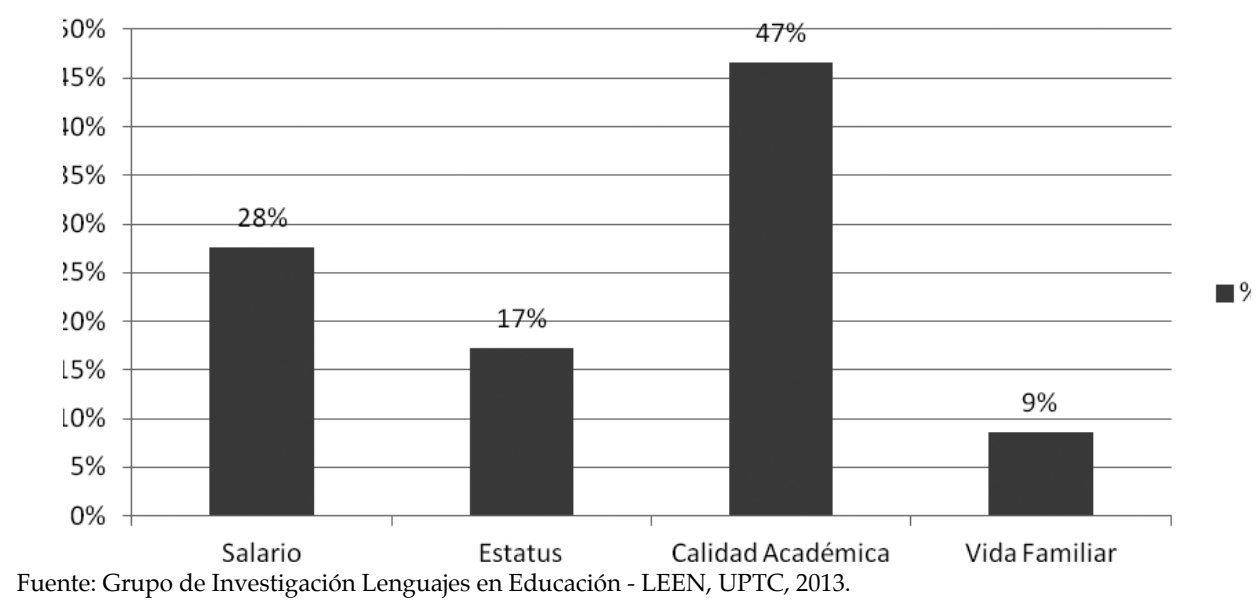




\section{Gráfica 5. Porcentaje del campo en que un profesional debe cursar educación posgraduada, según estudiantes de pregrado.}

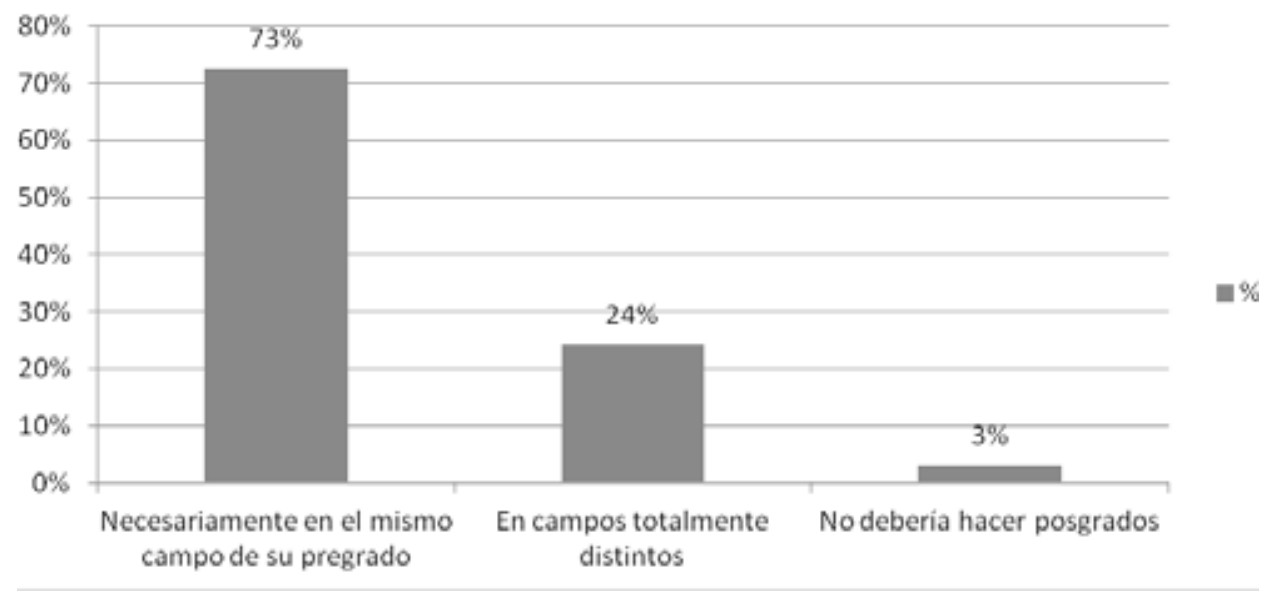

Fuente: Grupo de Investigación Lenguajes en Educación - LEEN, UPTC, 2013.

Como se observa en la gráfica 5, los estudiantes de pregrado perciben que al cursar un programa de posgrado sin duda mejorará su calidad de vida. Así mismo, la gráfica 5 demuestra que aún existe la idea de la disciplinariedad en el campo de estudio del pregrado, es decir, que un porcentaje alto de estudiantes del $73 \%$ reconoce que necesariamente se debe hacer posgrados en el mismo campo de estudio del pregrado, un $24 \%$ en campos totalmente distintos, y un porcentaje del $3 \%$ cree que no debería hacer posgrados.

Los estudiantes de pregrado sugieren que para mejorar la oferta de posgrados, por parte de la Facultad de Ciencias de la Educación de la Universidad Pedagógica y Tecnológica de Colombia, es necesario implementar una publicidad masiva, ampliar la oferta de maestrías y doctorados interdisciplinares, que apunten a la solución de los problemas sociales, que disminuyan los costos de matrícula, a la vez que se faciliten los mecanismos de ingreso. Así mismo, solicitan la oferta de becas y la facilidad de acceso para los estudiantes de las licenciaturas; promover a nivel interno las posibilidades que tienen académicamente al finalizar la licenciatura o antes; implementar posgrados en las áreas de música, artes o filosofía. 
Favorecer las condiciones de los programas de posgrado, tanto a nivel interno como externo, e internacionalizarlos y ofrecerlos en modalidad semestral y no solo anual.

En un tercer momento, se hace un análisis de reconocimiento de los programas de posgrado por parte de los estudiantes de postrado de la Facultad de Ciencias de la Educación de la UPTC. Por medio de una muestra aleatoria, se seleccionaron veinticinco estudiantes de los programas de posgrado, correspondientes al Doctorado en Historia, Doctorado en Lenguaje y Cultura, Maestría de la Cultura Física, Maestría en Educación, Maestría en Historia, Especialización en Gerencia Educacional. El objetivo de estas encuestas fue valorar las percepciones y la visibilidad que el nivel de posgrado tiene entre los estudiantes de postgrados de la Facultad de Ciencias de la Educación. Así, la variable independiente correspondió a estudiantes de posgrado de los primeros semestres para un total de veinticinco encuestados, como población objeto de estudio. El 100\% de las encuestas se aplicaron dentro de los espacios académicos de los programas o en oficinas administrativas donde concurren estudiantes de este nivel, como la Dirección de la Escuela de posgrados de la Facultad; lo cual favoreció las preguntas abiertas en relación con las respuestas dadas. Del total de encuestados, el $44 \%$ corresponde al género femenino y el $56 \%$ al género masculino, sin que se determine ningún porcentaje frente a otros géneros. Del total de encuestados, el $64 \%$ labora como docentes de educación básica y media, el $4 \%$ corresponde a un docente académico-administrativo en su cargo de decano, el $4 \%$, a un profesional que labora en la Universidad de Boyacá, otro $4 \%$ realiza actividades editoriales en una revista indexada de la Facultad y el $4 \%$ final no labora y solamente se dedica a estudiar. El 20\% del total de encuestados, no respondió la actividad profesional al momento de desarrollar la encuesta; sin embargo, se aclara que el $100 \%$ encuestado está realizando estudios de posgrado, a nivel de especialización, maestría o doctorado de la Facultad de Ciencias de la Educación.

Así mismo, se encontró que las empresas que acogen a los estudiantes son: $32 \%$ en la Universidad Pedagógica y Tecnológica de Colombia, el 28\% no respondió, el $8 \%$ en la Universidad de Boyacá, el $4 \%$ respectivamente en la Universidad Autónoma de Colombia, Universidad Distrital, Universidad Militar, Universidad Cooperativa de Colombia, Instituciones Educativas Rafael Uribe, Técnica de Nazaret, Ciudadela Sucre, Soacha, Sena. 
De igual manera, esta encuesta tuvo un rango entre los años 2000 a 2013 como la época en la cual se cursaron los programas de posgrado por parte de los encuestados. El 36\% manifestó haber cursado algún programa de posgrado pero no mencionó el año. Respectivamente, un $4 \%$ desarrolló cursos de posgrado en 2000, 2004, 2005 y 2007; el 8\%, respectivamente en 2010, 2011 y 2013; y un 12\% en el 2006 y 2012.

El $60 \%$ de los encuestados ha realizado estudios de maestría, tanto en la UPTC como en otras universidades; un $8 \%$, especialización; otro $8 \%$, un doctorado; el $24 \%$ no respondió, a pesar de ser estudiantes de posgrado. El $16 \%$ dejó inconcluso algún postgrado, el $72 \%$ ha finalizado sus estudios de posgrado, y el 12\% no respondió. El 16\% no concluyó sus estudios por las siguientes razones: la no realización del trabajo de grado o tesis doctoral, por asuntos familiares, por un factor económico o por crisis emocional.

En relación con la gráfica 6, el programa con mayor visibilidad fue la Maestría en Lingüística $17 \%$, seguida por la Maestría en Educación con un $15 \%$, luego el Doctorado en Historia con un 14\%, la Maestría en Historia con un $10 \%$ y los programas de Maestría en Literatura, Gerencia Educacional y el Doctorado en Lenguaje y Cultura con un $7 \%$, respectivamente; con un 5\% están los programas de Especialización en Archivística, Doctorado en Ciencias de la Educación, un 3\% lo obtuvo el Doctorado en Geografía y los programas en Derechos Humanos que no pertenecen a la Facultad, y un $2 \%$ para la Maestría en Docencia de los Idiomas, la Maestría en Geografía y Maestría en Pedagogía de la Cultura Física. Un 2\% dijo no conocer los programas. 


\section{Gráfica 6. Porcentaje reconocimiento de los programas de pos- grado de la Facultad de Ciencias de la Educación de la UPTC, según sus mismos estudiantes}

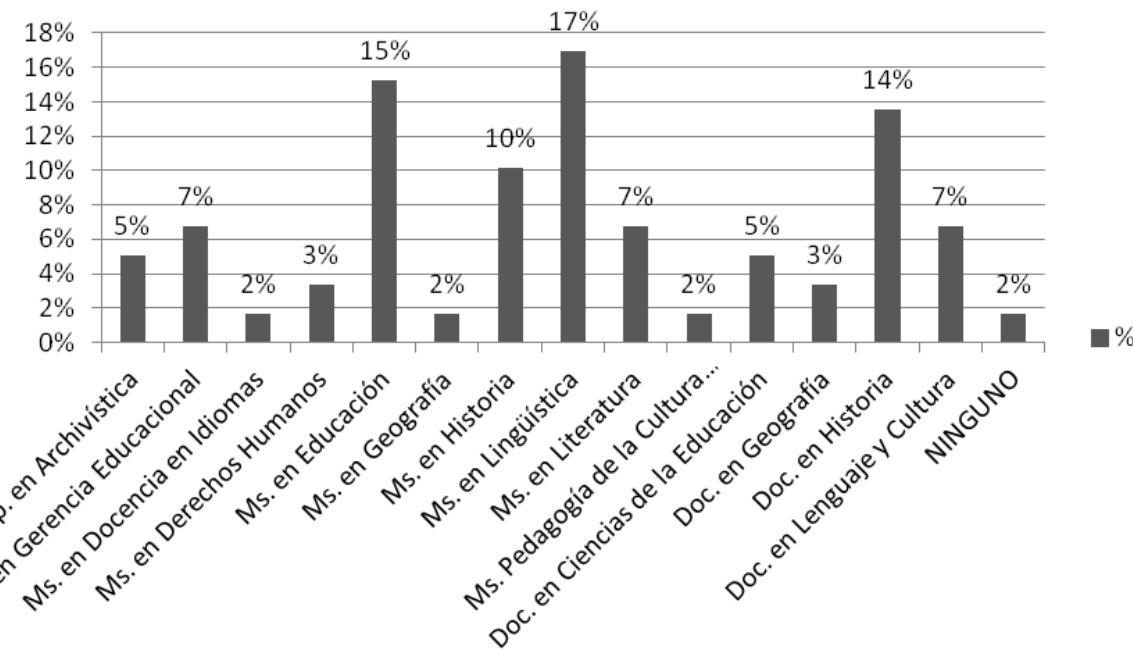

Fuente: Grupo de Investigación Lenguajes en Educación - LEEN, UPTC, 2013.

Como se observa en la Gráfica 6, los encuestados mencionaron programas que no corresponden a la Facultad de Ciencias de la Educación, pero que, por asociación al campo disciplinar, lo asumen como tal: Maestría en Derechos Humanos y Maestría en Economía. De igual manera, asimilan o relacionan las líneas de investigación con los programas en sí, como el Doctorado en historia de la educación; trasladan algunos programas de pregrado al programa de posgrado como la Maestría en Lengua Extranjera, que tiene que ver con el programa de pregrado denominado Licenciatura en Lengua Extranjera, en lugar de la Maestría en Docencia de Idiomas.

En relación con la gráfica 7 , el medio de comunicación por el cual se enteraron de los programas de postgrado, el $30 \%$, manifestó que por medio de un amigo y la web; el 15\% por ser estudiante o trabajador de la universidad; el $12 \%$, por medio de folletos; un $6 \%$ por medio de la radio, y un porcentaje reducido, es decir un $3 \%$, ha conocido los programas de posgrado por medio de un familiar o por la prensa. Dejando entonces evidente que ninguno de los estudiantes de posgrado encuentra información de los posgrados que ofrece la Facultad de Ciencias de la Educación en medios como la televisión o la valla. 
Gráfica 7. Porcentaje medios de información que dan a conocer los programas de posgrado de la Facultad de Ciencias de la Educación de la UPTC, según sus mismos estudiantes.

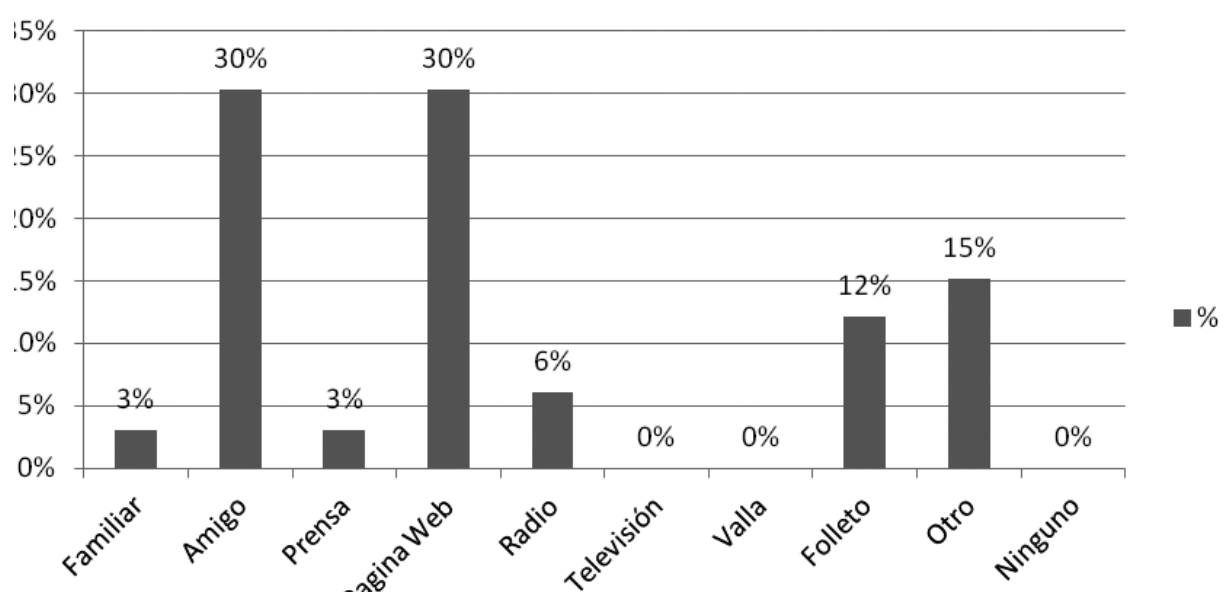

Fuente: Grupo de Investigación Lenguajes en Educación - LEEN, UPTC, 2013.

Gráfica 8. Porcentaje de impacto al cursar y finalizar un programa de posgrado de la Facultad de Ciencias de la Educación de la UPTC, según sus mismos estudiantes

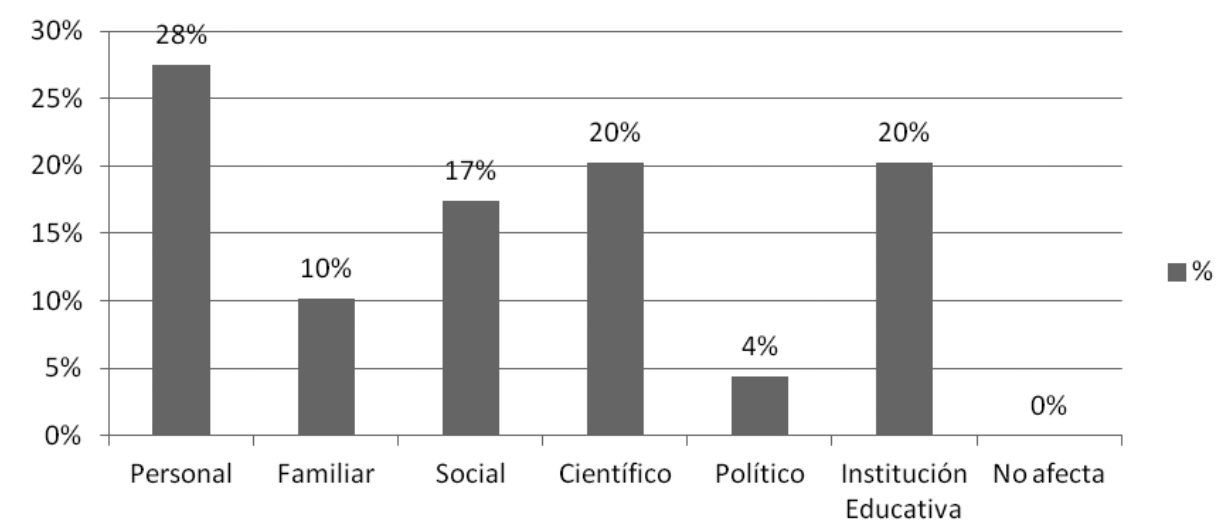

Fuente: Grupo de Investigación Lenguajes en Educación - LEEN, UPTC, 2013.

Como se observa en la gráfica 8, el campo que se ve impactado favorablemente al cursar y finalizar un programa de posgrado de la Facultad de Ciencias de 
la Educación de la UPTC es en un $28 \%$ el campo personal, en un $20 \%$ el científico y la institución educativa, en un $17 \%$ el social, para dejar con un $10 \%$ el familiar, y con un $4 \%$ el campo político.

\section{Gráfica 9. Porcentaje de los aspectos que mejorarán al cursar un programa de posgrado de la Facultad de Ciencias de la Edu- cación de la UPTC, según sus mismos estudiantes}

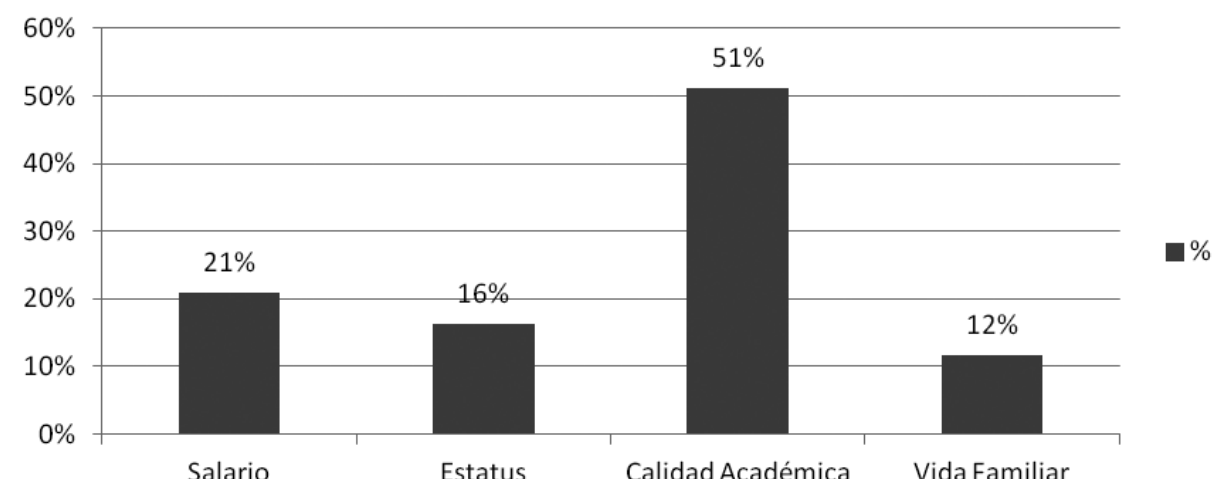

Fuente: Grupo de Investigación Lenguajes en Educación - LEEN, UPTC, 2013.

En relación con la gráfica 9, se observa que los estudiantes de posgrado en un $51 \%$ cree que quien cursa un programa de posgrado en la Facultad de Ciencias de la Educación de la UPTC, sin duda mejorará en su calidad académica, seguido de un $21 \%$ en su salario, para dejar con un $16 \%$ el estatus social y con un $12 \%$ la vida familiar.

De igual manera, en la gráfica 10, el 78\% de los encuestados manifestó que todo profesional debe cursar educación posgraduada en el mismo campo de su pregrado y un $22 \%$, en campos totalmente distintos.

Como se observa en la gráfica 11, el $54 \%$ de los encuestados manifestó que los programas de posgrado de la UPTC cumplen con sus expectativas suficientemente, y un $13 \%$ plenamente, para dejar un $33 \%$ como aceptablemente. 
Gráfica 10. Porcentaje del campo en que un profesional debe cursar educación posgraduada, según sus mismos estudiantes.

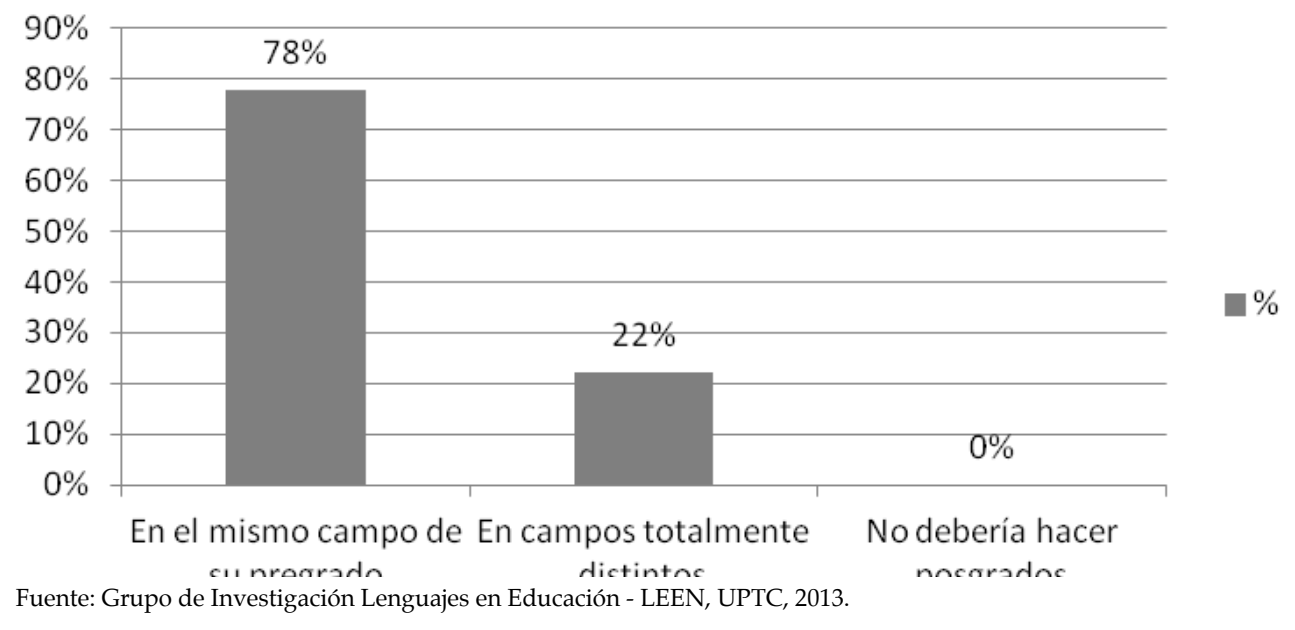

\section{Gráfica 11. Porcentaje del cumplimiento de expectativas de quienes cursan programas de posgrado de la Facultad de Cien- cias de la Educación de la UPTC}

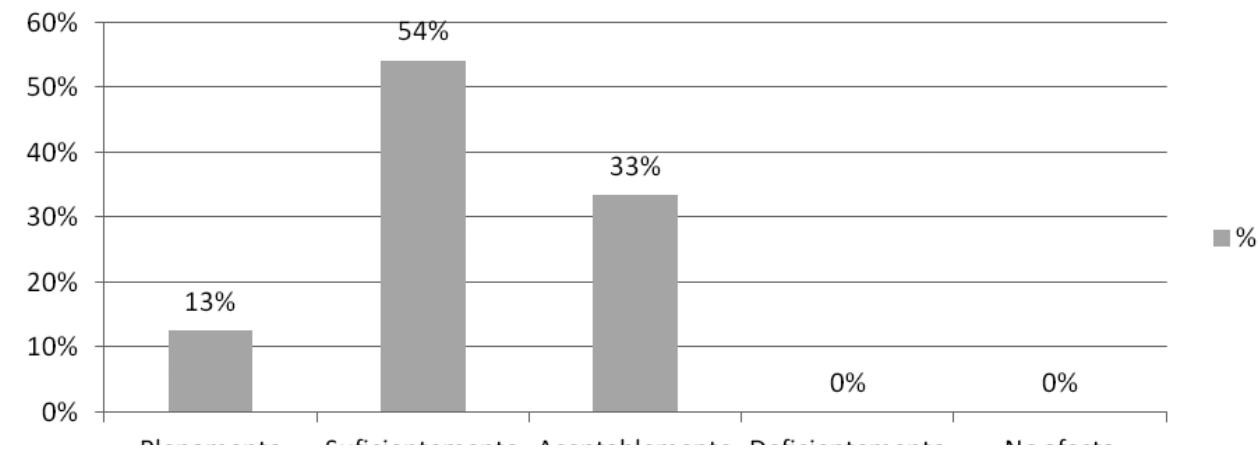

Fuente: Grupo de Investigación Lenguajes en Educación - LEEN, UPTC, 2013.

Algunas razones planteadas por los encuestados frente a este criterio, en relación con el ítem plenamente fueron, que existen profesores y currículos de calidad, además que hay docentes con mucha capacidad investigativa que aportan nuevos conocimientos e incentivan a fortalecer y ampliar las áreas que ellos manejan. 
En relación con el ítem suficientemente, manifestaron que hay diversidad de programas que permiten una integración del conocimiento y enriquecen los saberes propios de cada área, pero que, sin embargo, hay niveles académicos con muy baja calidad, donde se encuentra la improvización de seminarios y no hay grupos de investigación que puedan soportarlos. A pesar de lo anterior, un buen número cree que la totalidad del plan de estudios se relaciona suficientemente con las preocupaciones individuales, especialmente en el campo del Lenguaje y la Cultura. En otro sentido, se observó preocupación porque en algunos programas los profesores de pregrado repiten los contenidos, sin profundización a nivel de posgrado. De igual manera, sugieren precisión en relación con los horarios y materiales, otra queja recurrente fue la preocupación por los continuos bloqueos y paros que impiden el desarrollo de los procesos académicos.

En relación con el ítem aceptablemente, los encuestados manifestaron quedar solos, con muy bajo nivel de acompañamiento, en los procesos deinvestigación y profundización, especialmente durante el desarrollo de los trabajos de grado y tesis doctoral. Así mismo, observan que hay desorganización y desarticulación entre los programas de pregrado y posgrado; finalmente, manifiestan que, así como hay estudiantes excelentes, algunos no cumplen con esta característica a nivel de doctorado.

En relación con los procesos de Internacionalización, el 32\% de los encuestados identifica aspectos de la internacionalización, como se observa en la gráfica 12. Del $68 \%$ restante de los encuestados, $24 \%$ no conoce, $20 \%$ no sabe, y $24 \%$ no respondió, lo cual preocupa porque es un porcentaje muy alto de estudiantes que no conocen este campo en su proceso de formación.

Algunas razones sobre internacionalización que manifestaron el 32\% de los encuestados, fue la participación de conferencistas extranjeros, los convenios de la UPTC con otras instituciones que les posibilitaron realizar pasantías, el contacto con otros investigadores, la escritura de artículos en revistas indexadas, la participación en simposios y congresos internacionales, organizados tanto por la UPTC, como por las universidades externas. El 68\% de los encuestados entiende que el posgrado genera un espacio internacional en la medida que cuenta con profesores de universidades internacionales. Así, manifiestan que existe poca información y divulgación de los convenios y pasantías, a los que pueden acogerse. Algunos no saben sobre los procesos 
de internacionalización, ni identifican la oficina de relaciones internacionales, ni las posibilidades que les ofrece como estudiantes de posgrado, en razón a la poca visibilidad y socialización de dichos procesos.

\section{Gráfica 12. Porcentaje de identificación de procesos de interna- cionalización en la experiencia posgraduada de los estudiantes UPTC}

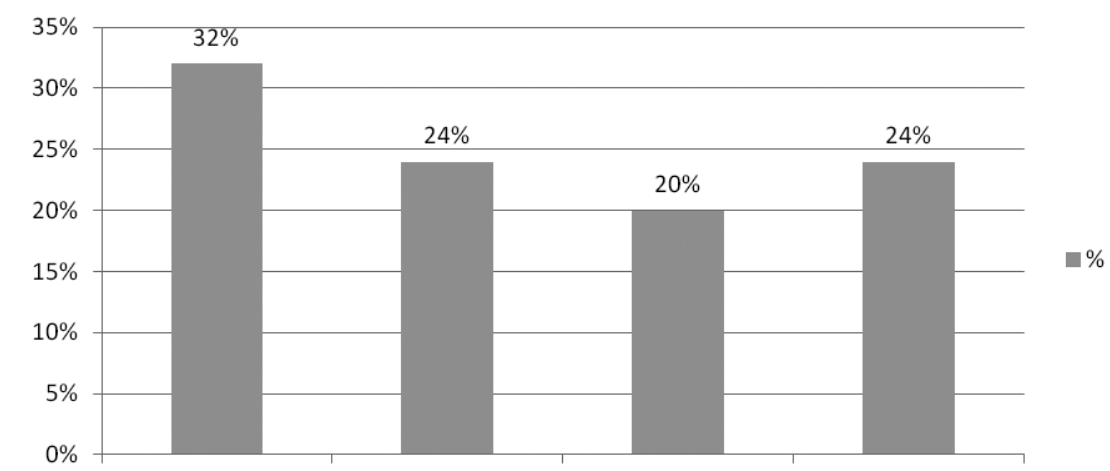

Fuente: Grupo de Investigación Lenguajes en Educación - LEEN, UPTC, 2013.

Finalmente, los encuestados sugieren que para mejorar la oferta de posgrados por parte de la Facultad de Ciencias de la Educación de la Universidad Pedagógica y Tecnológica de Colombia, se debe exigir un mayor rigor académico y científico, que funcionen de manera institucional y no con "dueños" que convierten a los posgrados en una feria de la educación; que se realicen eventos académicos que integren a varios programas de posgrado y varias áreas de conocimiento; que se actualicen los procesos administrativos para este nivel, dado que son obsoletos, poco dinámicos y funcionales. Los anteriores, son algunos de los problemas graves, con los que se cuenta en estos momentos, así como garantizar los recursos financieros y logísticos para un adecuado desarrollo, junto con la realización de estudios de factibilidad en concordancia con las necesidades de formación universitaria y de formación continuada, tanto para los profesores de la UPTC, como de los profesores que trabajan en los niveles de Educación Básica Primaria, Media.

En relación con la investigación, se sugiere hacer un seguimiento riguroso con profesores externos a la universidad, puesto que en ocasiones, profesores 
y temáticas, son los mismos en el pregrado, lo cual quita credibilidad al programa. De igual manera, se sugiere conformar comités curriculares que trabajen a nivel de doctorado y maestrías; así mismo, se cuestiona el hecho de que al ser el posgrado una profundización en un campo de conocimiento no se debe tomar como una opción para hacer un semestre y graduarse del pregrado. En relación con lo pedagógico, se propone hacer más evidente la misión de la facultad, desde una perspectiva básica que permita hacer simple lo complejo. En relación con la visibilidad se sugiere una mejor divulgación de los programas de posgrado con la modalidad, dedicación y tiempo, en todos los medios masivos de comunicación y no solo en la página web de la Universidad. Así mismo, se sugiere dar a conocer los programas en las capacitaciones del Ministerio de Educación Nacional, con los docentes que laboran en la universidad como en las instituciones educativas, entre otras.

\section{CONCLUSIÓN}

La Facultad de Ciencias de la Educación de la UPTC ha realizado procesos que le permiten consolidarse como una Facultad con programas de pregrado, que impactan significativamente en la formación de docentes en el ámbito regional. Sin embargo, la educación posgraduada no forma parte del discurso educativo, en la misma medida que lo hacen los programas de pregrado.

Las políticas del Estado desde el siglo XIX han buscado fortalecer los niveles básicos y secundarios, dejando de lado la Educación Superior y a merced de las dinámicas regionales y descontextualizadas de la educación en general. Solo desde la Ley 80 del 1980, se incorpora la investigación como un componente fundamental en la Educación Superior, frente a los avances sustanciales de la Educación Superior de carácter privado. La Educación Superior Pública debe elaborar políticas que le permitan, por una parte, mejorar la cobertura y fortalecer la calidad, y, por la otra, implementar la práctica educativa con investigación.

Hoy por hoy, los avances logrados como resultados de investigación, en pocas ocasiones trascienden a los demás niveles educativos. El sistema se convierte en un proceso desarticulado que no le permite participar en un proceso de construcción de ciudadanía. 
Sorprende ver dos aspectos en el análisis de los resultados encontrados en esta investigación: por una parte, si se tiene en cuenta que las políticas de los posgrados se consolidan a nivel normativo desde la Ley 80 de 1980, sorprende encontrar que la UPTC solamente haya dispuesto de una reglamentación posgraduada diecinueve años después. Por otro lado, no obstante la reglamentación dispuesta en el acuerdo 108 de 1999, el cual tuvo una vigencia de 13 años, y luego con los Acuerdos 025 de 2012 y 052 de 2012, continúen las políticas educativas en torno de la formación de pregrados, tan es así, que no se cuenta con un sistema de información y control propio de los programas de posgrados. Las descargas académicas se hacen a partir de los pregrados y los posgrados se encuentran sometidos en todo y cada uno de sus aspectos de calidad, cobertura y eficiencia, alrededor de los pregrados. Es imperante generar en los posgrados la autonomía necesaria para que se conviertan en la punta de lanza del desarrollo de la Universidad y de la sociedad. Actualmente, el nivel posgraduado compromete a una gran masa de estudiantes, quienes, en varias ocasiones, transfieren las lógicas de los pregrados, dado que se carece de una estructura adecuada que rompa con los imaginarios resultantes de una concepción profesionalizante.

Así mismo, se observa que un problema álgido es la asignación de actividad docente a los pregrados, antes que los programas de posgrados. Para la actividad administrativa eventualmente se dispone de la asignación académica, pero para los posgrados no. Este aspecto, contrario al espíritu de la norma, continua vigente. Por lo tanto, se hace necesario favorecer este nivel con políticas de investigación, publicación e internacionalización.

\section{FUENTES}

Consejo Nacional de Acreditación - CNA. (2009). Lineamientos para la Acreditación de Alta Calidad de programas de Maestría y Doctorado. Ciudad: Ministerio de Educación, Año.

Resoluciones 16 de 2009, 49 de 2009.

Universidad Pedagógica y Tecnológica de Colombia. Acuerdos: 108 de 1999, 025 de 2012, 052 de 2012, 028 de 2010, 052 de 2011, 053 de 2011, 35 de 2011, 02 de 2012, 06 de 2012. 012 de 1999. 


\section{REFERENCIAS}

Acuña Rodríguez, Olga Yanet. "Tendencias historiográficas vistas a través de las tesis del Doctorado en Ciencias de la Educación RUDECOLOMBIA (1988-2007)". Revista Historia de la Educación Latinoamericana 12 (2009): 79-95.

De Flachs Vera, NOMBRE. "Las primeras mujeres universitarias en Córdoba (Argentina) y la Escuela de Parteras". Revista Historia de la Educación Latinoamericana 3 (2001): 135-166.

Lucena, Manuel. "La universidad colonial hispanoamericana de Carlos IV". Revista historia de la Educación Latinoamericana 3 (2001): 47-68

Morales, Víctor. Educación, Poder y Futuro. Caracas: Universidad Central de Venezuela, Facultad de Humanidades y Educación, Escuela de Educación, 1988.

Soto Arango, Diana. "El profesor universitario de América Latina: Hacia una responsabilidad ética, científica y social." Revista Historia de la Educación Latinoamericana 13 (2009): 166-188.

Soto Arango, Diana. "La calidad y la evaluación de la educación en los estudios superiores en la Audiencia de Santa Fe". Revista Historia de la Educación Latinoamericana 1 (1998): 113-127.

Soto Arango, Diana. "La ruralidad en la cotidianidad escolar colombiana. Historia de vida de la maestra rural boyacense, 1948-1980". Revista Historia de la Educación Latinoamericana18 (2012): 211-242.

Torres Cruz, Doris Lilia. "Textos y pedagogías en los albores del siglo XX en Colombia”. Revista Historia de la Educación Latinoamericana 16 (2011): 255-278.

Torres Cruz, Doris Lilia. "Postgrados en Iberoamérica, historia y prospectiva.". VIII Congreso Internacional de la Sociedad de Historia de la Educación Latinoamericana. III Seminario Taller Internacional Vendimia 2009 "La Construcción de Nación en Latinoamérica Siglos XIX al XXI: Comparaciones y Conexiones", 50-65, v.16, fasc.16.Universidad Pedagógica y Tecnológica de Colombia UPTC, 2011.

Torres Cruz, Doris Lilia. “La formación postgraduada hoy”. XIII Congreso Solar. Colombia. 2012.

Van Dijk, Teun A. "Ideología y análisis del discurso". Revista Utopía y Praxis Latinoamericana 10 (2005): 9.

\begin{tabular}{|l|} 
\\
\hline Torres Cruz, Doris Lilia y Prieto Ortega, Jinny Fernanda. “Posgra- \\
dos: visión y percepción en la Universidad Pedagógica y Tecnológica \\
de Colombia (UPTC)". Revista Historia de la Educación Latinoameri- \\
cana. Vol. 16 No, 22, (2014): 249 - 273.
\end{tabular}

